\title{
Una nueva función de densidad simétrica
}

\author{
A New Symmetric Density Function
}

José Alfredo Jiménez Moscosda

josajimenezm@unal.edu.co

\section{Resumen}

En este artículo se presenta una nueva función de densidad simétrica que tiene colas más pesadas que la distribución normal. Se determina la media y la varianza de esta distribución, lo cual permite estimar los parámetros por el método de los momentos.

Palabras clave: cosecante hiperbólica, colas pesadas.

\begin{abstract}
This paper present a new function symmetrical density that presented heavy tails. Also, the mean and the variance associated to this distribution are determined that allows to estimate the parameters by the method of the moments.
\end{abstract}

Key words: Hyperbolic Cosecant, Heavy Tails.

\section{Introducción}

En Johnson \& Kotz (1970) y Stuart \& Ord (1994), se presenta una gran variedad de funciones de densidad; sin embargo, en muchas situaciones la distribución de la población es desconocida y, por tanto, entre más alternativas de distribuciones se tengan le proporcionará al investigador más herramientas para su estudio.

En este artículo se presenta una nueva función de densidad que tiene colas pesadas y además se presentan tabulados los valores de la función de distribución. En los apéndices aparecen los detalles de los resultados presentados.

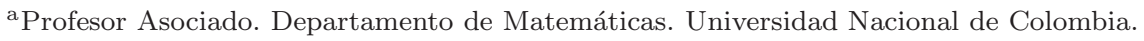




\section{Función de densidad}

Una variable aleatoria continua $X$ tiene distribución cosecante hiperbólica si su función de densidad está dada por

$$
f_{X}(x)=f_{X}(x ; \mu, \beta)= \begin{cases}\frac{\lambda^{2}}{\beta}\left(\frac{x-\mu}{\beta}\right) \operatorname{csch}\left(\frac{x-\mu}{\beta}\right), & x \neq \mu \\ \frac{\lambda^{2}}{\beta}, & x=\mu .\end{cases}
$$

con $x \in \mathbb{R}, \mu \in \mathbb{R}, \beta>0$ y $\lambda=\frac{\sqrt{2}}{\pi}$. La forma (curva) simple, se obtiene haciendo el cambio de variable $z=\frac{x-\mu}{\beta}$ y se llega a

$$
f_{Z}(z)=f_{Z}(z ; 0,1)= \begin{cases}\lambda^{2} z \operatorname{csch}(z), & z \neq 0 \\ \lambda^{2}, & z=0\end{cases}
$$

En la Figura 1 1 se muestran varias gráficas de la función de densidad dada en (2.1) para cinco valores de $\beta$ y $\mu=0$.

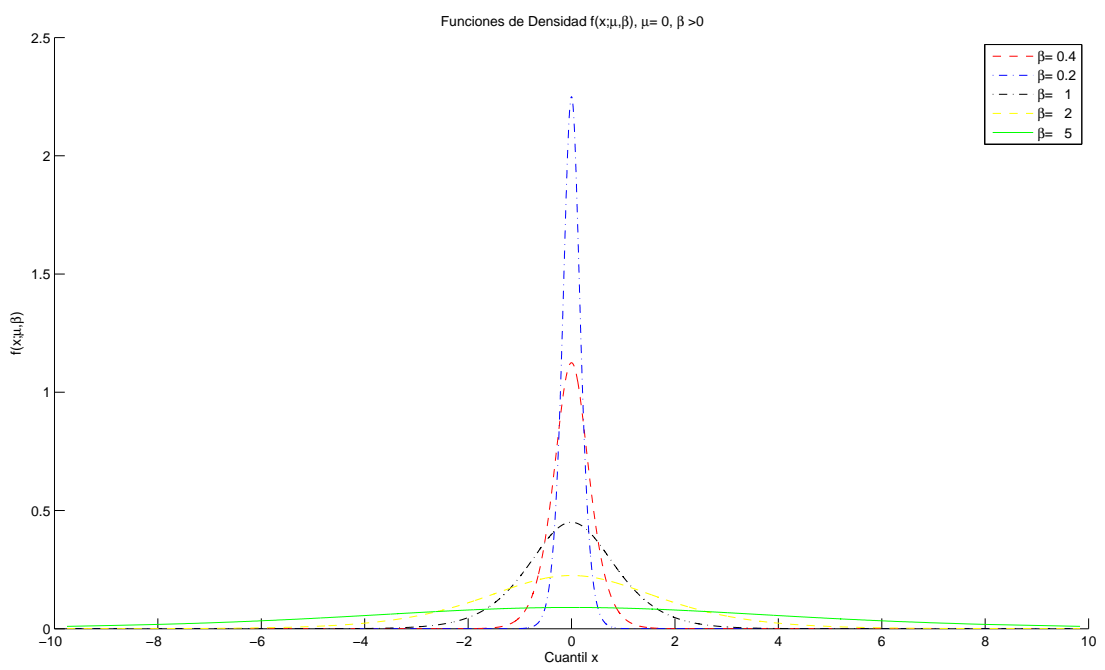

Figura 1: Comparación de funciones de densidad con $\mu=0$ y $\beta$ arbitrario 


\subsection{Función de distribución}

La probabilidad $P(X \leq x)$, viene dada por

$$
\begin{gathered}
F_{X}(x ; \mu, \beta)=P(X \leq x)=\frac{2}{\beta \pi^{2}} \int_{-\infty}^{x} \frac{u-\mu}{\beta} \operatorname{csch}\left(\frac{u-\mu}{\beta}\right) d u \\
=1-\frac{1}{\pi^{2}}\left\{4 \operatorname{Li}_{2}\left(\tanh \left(\frac{1}{2} \frac{x-\mu}{\beta}\right)\right)-\operatorname{Li}_{2}\left(\tanh ^{2}\left(\frac{1}{2} \frac{x-\mu}{\beta}\right)\right)\right. \\
\left.\quad-2 \frac{x-\mu}{\beta} \ln \left(\tanh \left(\frac{1}{2} \frac{x-\mu}{\beta}\right)\right)\right\}
\end{gathered}
$$

donde $\operatorname{Li}_{2}(\cdot)$ denota la función dilogarítmica, en Abramowitz \& Stegun (1965) aparece tabulada esta función. Aunque la integral en (2.3) se pudo evaluar de manera cerrada, los valores de la función dilogarítmica no son fáciles de obtener; por lo tanto, se puede tabular $F_{X}(x ; \mu, \beta)$ como una función de $\mu \mathrm{y} \beta$. Sin embargo, se necesitaría una tabla para cada pareja de valores. Puesto que existe un número infinito de valores de $\mu$ y $\beta$, este procedimiento es numéricamente imposible. Este problema se puede solucionar empleando la siguiente sustitución $z^{*}=\lambda \frac{x-\mu}{\beta}$ y la función $F_{Z^{*}}\left(z^{*} ; 0,1\right)$ se encuentra tabulada de manera extensa en el Apéndice C. Debido a la simetría se verifica que

$$
P\left(Z^{*} \leq-z^{*}\right)=1-P\left(Z^{*} \leq z^{*}\right) .
$$

En la Tabla 1 1se comparan los valores de algunas funciones de distribución asociadas a funciones de densidad simétricas (Logística, Laplace, Normal y la nueva).

Como puede notarse la probabilidad en la cola derecha a una distancia de 5 desviaciones estándar de la media es todavía distinta de uno.

Teorema 2.1. La función $f_{X}(x)$ dada en (2.1) satisface las siguientes propiedades:

1. Es continua para todo $x \in \mathbb{R}$.

2. Tiene un máximo en $x=\mu$.

3. Es simétrica respecto a $\mu$.

4. El eje $X$ es asíntota horizontal de la función.

Teorema 2.2. Los momentos centrales de orden $k$ de una variable aleatoria $X$ con función de densidad dada por la expresión (2.1) vienen dados por

$$
\mu_{k}=\left\{\begin{array}{lll}
2(-1)^{m} \frac{2^{2(m+1)}-1}{m+1}(\beta \pi)^{2 m} B_{2(m+1)} & \text { si } & k=2 m \\
0 & \text { si } & k=2 m+1,
\end{array}\right.
$$

donde $\mu_{k}=E\left[(X-\mu)^{k}\right]$ y $B_{k}$ denota el k-ésimo número de Bernoulli. 


\begin{tabular}{r|rrrr}
\hline$z^{*}$ & Nueva & Logística & Laplace & Normal \\
\hline 0.0 & 0.5000 & 0.5000 & 0.5000 & 0.5000 \\
0.2 & 0.5891 & 0.5897 & 0.6232 & 0.5793 \\
0.4 & 0.6726 & 0.6738 & 0.7160 & 0.6554 \\
0.6 & 0.7464 & 0.7481 & 0.7860 & 0.7257 \\
0.8 & 0.8082 & 0.8102 & 0.8387 & 0.7881 \\
1.0 & 0.8580 & 0.8598 & 0.8784 & 0.8413 \\
1.2 & 0.8965 & 0.8981 & 0.9084 & 0.8849 \\
1.4 & 0.9257 & 0.9269 & 0.9310 & 0.9192 \\
1.6 & 0.9472 & 0.9479 & 0.9480 & 0.9452 \\
1.8 & 0.9628 & 0.9632 & 0.9608 & 0.9641 \\
2.0 & 0.9741 & 0.9741 & 0.9704 & 0.9772 \\
2.2 & 0.9820 & 0.9818 & 0.9777 & 0.9861 \\
2.4 & 0.9876 & 0.9873 & 0.9832 & 0.9918 \\
2.6 & 0.9915 & 0.9911 & 0.9874 & 0.9953 \\
2.8 & 0.9942 & 0.9938 & 0.9905 & 0.9974 \\
3.0 & 0.9960 & 0.9957 & 0.9928 & 0.9987 \\
3.2 & 0.9973 & 0.9970 & 0.9946 & 0.9993 \\
3.4 & 0.9982 & 0.9979 & 0.9959 & 0.9997 \\
3.6 & 0.9988 & 0.9985 & 0.9969 & 0.9998 \\
3.8 & 0.9992 & 0.9990 & 0.9977 & 0.9999 \\
4.0 & 0.9994 & 0.9993 & 0.9983 & 1.0000 \\
4.2 & 0.9996 & 0.9995 & 0.9987 & \\
4.4 & 0.9998 & 0.9997 & 0.9990 & \\
4.6 & 0.9998 & 0.9998 & 0.9993 & \\
4.8 & 0.9999 & 0.9998 & 0.9994 & \\
5.0 & 0.9999 & 0.9999 & 0.9996 & \\
\hline \hline
\end{tabular}

* Denota múltiplos de desviaciones estándar

Tabla 1: Valores de funciones de distribución 


\section{Observaciones}

1. El valor esperado es

$$
E(X)=\mu .
$$

2. La varianza se obtiene reemplazando en (2.4) por $k=2,(m=1)$

$$
\mu_{2}=E\left[(X-\mu)^{2}\right]=\frac{1}{2}(\beta \pi)^{2} .
$$

3. El cuarto momento central viene dado por

$$
\mu_{4}=E\left[(X-\mu)^{4}\right]=(\beta \pi)^{4} .
$$

4. El coeficiente de curtosis sería

$$
\alpha_{4}=\frac{\mu_{4}}{\operatorname{Var}(X)^{2}}=\frac{E\left[(X-\mu)^{4}\right]}{E\left[(X-\mu)^{2}\right]^{2}}=4,
$$

luego, el exceso de curtosis de esta variable es positivo y por consiguiente su distribución tiene colas más pesadas que las de una distribución normal.

\subsection{Características}

1. La curva tiene un solo máximo, por tanto es unimodal y tiene forma de campana.

2. La media de una población distribuida de esta manera cae en el centro de su curva.

3. Debido a la simetría de la distribución, la mediana y la moda se encuentran también en el centro; en consecuencia, para esta curva, la media, la mediana y la moda tienen el mismo valor.

4. Los dos extremos de la distribución de probabilidad considerada se extienden indefinidamente y nunca tocan el eje horizontal, por lo que existe algo de probabilidad de que la variable aleatoria pueda tomar valores demasiado grandes o muy pequeños; sin embargo, no se pierde mucha precisión si se ignoran valores muy alejados de la media.

5. Si la distribución de la población de una variable es aproximada mediante esta distribución, entonces:

a) Alrededor del 71, $59 \%$ de los valores se encuentran a 1 desviación estándar de la media.

b) Alrededor del $94,81 \%$ de los valores se encuentran a 2 desviaciones estándar de la media.

c) Alrededor del 99,21\% de los valores se encuentran a 3 desviaciones estándar de la media. 


\section{Ejemplo}

Se analiza la divisa correspondiente al cambio Dólar/Euro (2261 observaciones entre enero de 1999 y octubre de 2007)1.

En la Tabla 2 se presentan los estadísticos de los retornos de la serie.

\begin{tabular}{lr}
\hline \multicolumn{2}{c}{ Estadísticos } \\
\hline \hline Media (\%) & 0.0088698 \\
Mínimo (\%) & -2.2520226 \\
Máximo (\%) & 4.2041335 \\
Desv. Est. (\%) & 0.6185473 \\
Asimetría & 0.2606829 \\
Curtosis & 4.5477208 \\
JB & 251.2783180 \\
\hline
\end{tabular}

Tabla 2: Resumen de Estadisticas

Claramente se nota que los retornos de la serie tienen mayor apuntamiento (exceso de curtosis). Este mayor apuntamiento implica una mayor probabilidad para pequeños movimientos en las colas de lo que se esperaría en una distribución normal, Gao (2009).

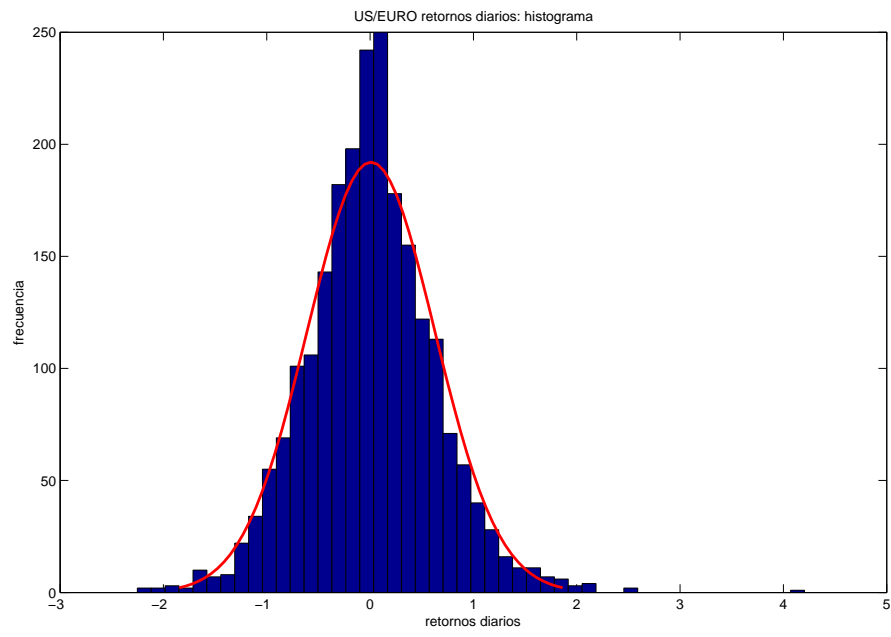

Figura 2: Histograma de frecuencias

\footnotetext{
${ }^{1}$ La fuente de información estadística para estas tasas de cambio es Federal Reserve Statistical Release http://www.federalreserve.gov/releases/h10/Hist.
}

Comunicaciones en Estadística, diciembre 2010, Vol. 3, No. 2 
La curtosis, asimetría y el test propuesto por Jarque \& Bera (1987)2, estadísticos reportados en la Tabla 2 indican que la hipótesis nula de una distribución normal puede ser rechazada para la variable en estudio. El histograma de frecuencias (Figura 2) también muestra que los retornos de la serie tienen un leve grado de sesgo a la derecha, es leptocúrtica y no sigue la distribución normal.

En la Figura 3 se muestra el nivel del precio y los retornos en el período muestral para la serie considerada.
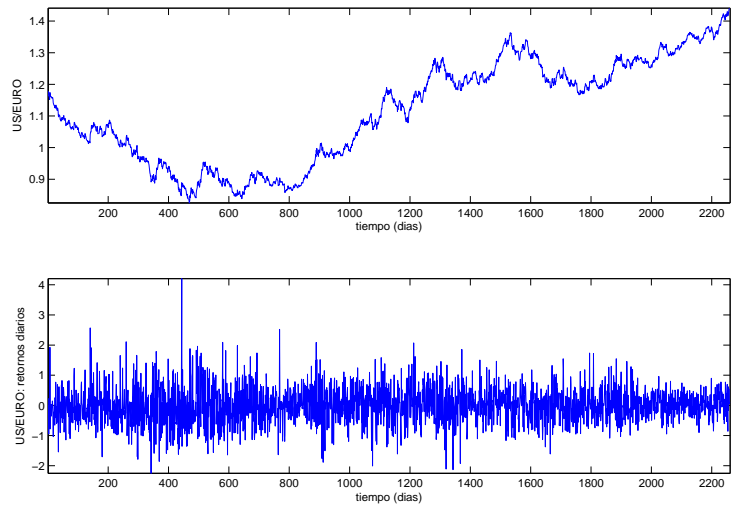

Figura 3: Nivel de Precio y retornos sobre el USEURO

Para determinar la forma de la distribución de la tasa de retorno se ajusta inicialmente el siguiente modelo de mixtura de normales

$$
f_{X}(x ; \boldsymbol{\Lambda})=\omega_{1} \varphi\left(x, \mu_{1}, \beta_{1}^{2}\right)+\omega_{2} \varphi\left(x, \mu_{2}, \beta_{2}^{2}\right)
$$

esta suma ponderada de densidades normales se expresa por $X \sim N M I X(\boldsymbol{\Lambda})$, donde $\boldsymbol{\Lambda}=\left(\xi_{\mathbf{1}}, \xi_{\mathbf{2}}\right)$ con $\xi_{\mathbf{j}}=\left(\mu_{j}, \beta_{j}^{2}, \omega_{j}\right)$ para $j=1,2 ; \varphi\left(x, \mu_{j}, \beta_{j}^{2}\right)$ denota la densidad de una variable aleatoria normal univariada con media $\mu_{j}$ y varianza $\beta_{j}^{2}$; las ponderaciones satisfacen que $\omega_{1}+\omega_{2}=1$. En este caso, se obtienen los parámetros reportados en la tabla 3, cuyas estimaciones fueron obtenidas mediante el método de momentos.

Para ajustar la nueva distribución se emplean los siguientes parámetros

$$
\mu=\bar{x} \quad \text { y } \quad \beta=\frac{\sqrt{2}}{\pi} S_{x},
$$

\footnotetext{
${ }^{2}$ Estadístico: $J B=\frac{N}{24}\left(\left(2 \alpha_{3}\right)^{2}+\left(\alpha_{4}-3\right)^{2}\right) \sim \chi_{(2)}^{2}$

$N$ : Número de observaciones. $\alpha_{3}$ : Coeficiente de asimetría.

$\alpha_{4}$ : Coeficiente de kurtosis.

Bajo la hipótesis nula de normalidad, el estadístico de Jarque-Bera está distribuido como una chi-cuadrado con 2 grados de libertad.
} 


\begin{tabular}{lr}
\hline \multicolumn{2}{c}{ Estimaciones de Parámetros } \\
\hline \hline$\mu_{1}$ & -0.02261234 \\
$\beta_{1}$ & 0.50903488 \\
$\mu_{2}$ & 0.12076403 \\
$\beta_{2}$ & 0.89704725 \\
$\omega_{1}$ & 0.78044125 \\
\hline
\end{tabular}

Tabla 3: Estimaciones para el ajuste de la NMIX $(\mathbf{\Lambda})$

donde $\bar{x}, S_{x}$ son la media y desviación estándar de la serie USEURO, respectivamente.

En la Figura 4 se presentan la distribución normal con parámetros media y desviación estándar iguales a las de la serie USEURO, una aproximación de la densidad de probabilidad de la serie3, la mixtura de normales y la nueva distribución.

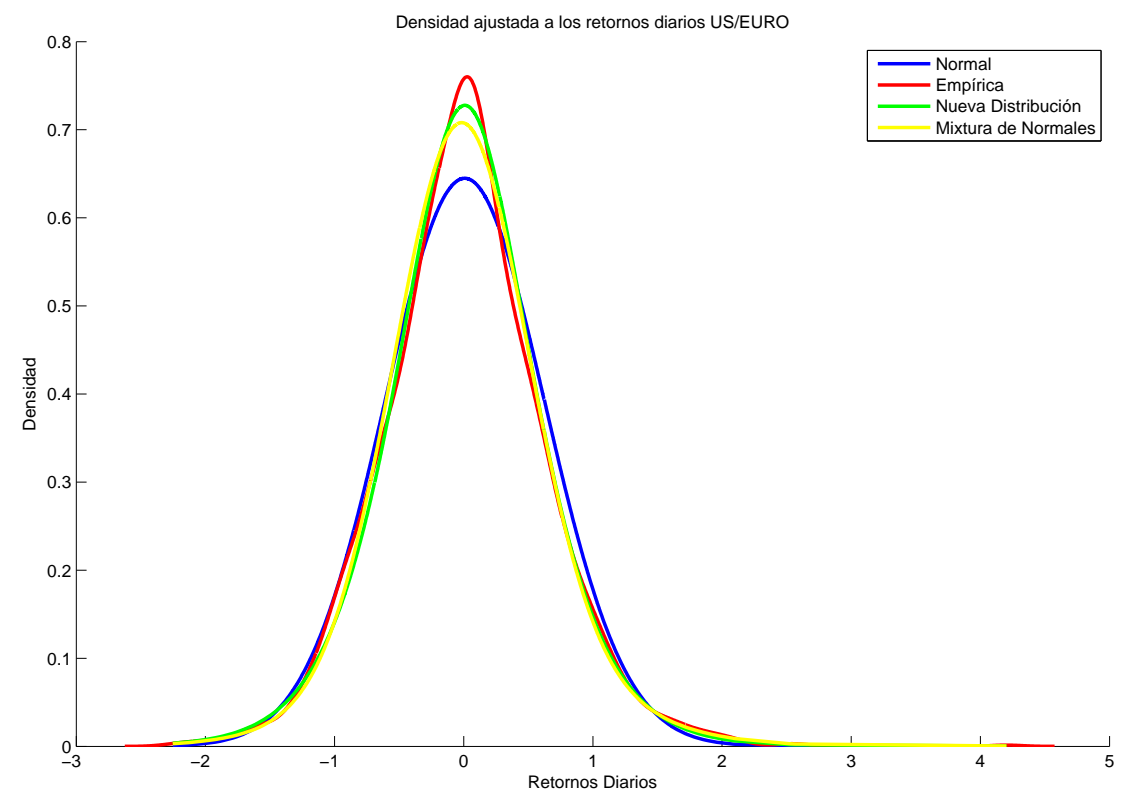

Figura 4: Comparación de densidades ajustadas

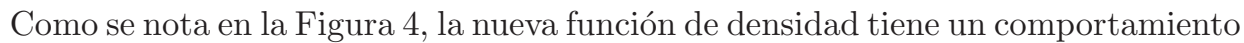
similar al histograma de los datos históricos de la variable de estudio presentada en la Figura 2 mientras que la distribución normal es la que presenta menos ajuste a los datos históricos.

\footnotetext{
${ }^{3}$ Esta densidad es obtenida con la instrucción "ksdensity" del programa MATLAB.
} 
En la Tabla 4 se presentan las siguientes medidas: media, dispersión, asimetría y curtosis, las cuales fueron obtenidas para cada una de las distribuciones ajustadas,

\begin{tabular}{|l|ccc|}
\hline Estadísticos & Serie & $N M I X(\boldsymbol{\Lambda})$ & Nueva densidad \\
\hline Media (\%) & 0.0088698 & 0.0104024 & 0.0088698 \\
Desv. Est. (\%) & 0.6185473 & 0.6190680 & 0.6185473 \\
Asimetría & 0.2606829 & 0.1578276 & 0.0000000 \\
Curtosis & 4.5477208 & 4.0322016 & 4.0000000 \\
\hline \hline
\end{tabular}

Tabla 4: Comparación de Estadísticas

Se obtiene que la media y la desviación estándar de la distribución nueva (ajustada con los parámetros dados en (3.1) ) son iguales que los de la serie de retornos, la mixtura de normales ajusta mejor la asimetría pero como se mostró, para ajustarla se necesita estimar cinco parámetros.

\section{Conclusiones}

En este artículo se obtiene una nueva función de densidad simétrica cuyos parámetros (dos en total) se pueden establecer de manera sencilla mediante el método de los momentos. Se establecieron todos los momentos centrales de forma explícita. Esta densidad tiene coeficiente de curtosis igual a 4. Aunque la expresión algebraica que la define es un poco complicada puede ajustarse a un conjunto de datos de manera más sencilla que la mixtura de normales para la cual se necesita estimar cinco parámetros.

Los desarrollos teóricos obtenidos en este artículo, permiten hacer uso de esta distribución en un amplio campo de acción, tanto en el cálculo de probabilidades a partir de la función de distribución como en la descripción gráfica y analítica de la función de densidad.

\section{Agradecimientos}

El autor agradece los valiosos comentarios y sugerencias de los árbitros anónimos de la Revista Comunicaciones en Estadística a la versión preliminar de este trabajo, los cuales ayudaron a clarificar la versión actual.

Recibido: 8 de mayo de 2010 Aceptado: 27 de septiembre de 2010 


\section{Referencias}

Abramowitz, M. \& Stegun, I. (1965), Handbook of Mathematical Functions, with Formulas, Graphs and Mathematical Tables, Dover Publications, Inc., New York.

Chew, V. (1968), 'Some useful alternatives to the normal distribution', The American Statistician 22(3), 22-24.

Gao, Q. (2009), Three Essays on Bio-security, Doctoral thesis, Texas A \& M University, Department of Agricultural Economics, Texas.

Jarque, C. M. \& Bera, A. K. (1987), 'A test for normality of observations and regression residuals', International Statistical Review 55(2), 163-172.

Johnson, N. L. \& Kotz, S. (1970), Distributions in Statistics: Continuous Univariate Distributions, Vol. 1,2, John Wiley \& Sons, Inc., New York.

Spiegel, M., Lipschutz, S. \& Liu, J. (2008), Schaum's Outline of Mathematical Handbook of Formulas and Tables, third edn, McGraw-Hill Professional, USA.

Stuart, A. \& Ord, J. K. (1994), Kendall's advanced theory of statistics. Vol. 1. Distribution theory, 6th edn, London : Edward Arnold, New York : Halsted Press.

\section{Apéndice}

En este apartado se verifica que la función dada en (2.1) es una función de densidad, para ello se usan las siguientes expresiones

$$
\begin{aligned}
& \sinh x=2 \sinh \left(\frac{1}{2} x\right) \cosh \left(\frac{1}{2} x\right) \\
& \cosh ^{2}\left(\frac{1}{2} x\right)-\sinh ^{2}\left(\frac{1}{2} x\right)=1 .
\end{aligned}
$$

Luego

$$
I=2\left(\frac{1}{\beta \pi}\right)^{2} \int_{-\infty}^{\infty}(u-\mu) \operatorname{csch}\left(\frac{1}{\beta}(u-\mu)\right) d u
$$

puesto que $\beta>0$, haciendo

$$
z=\frac{1}{\beta}(u-\mu) \rightarrow d z=\frac{1}{\beta} d u
$$

se llega a

$$
I=\frac{2}{\pi^{2}} \int_{-\infty}^{\infty} z \operatorname{csch}(z) d z=\frac{2}{\pi^{2}} \int_{-\infty}^{\infty} \frac{z}{\sinh z} d z
$$


la última función que aparece en la integral como ya se dijo representa la forma simple, entonces por la propiedad de simetría respecto al origen, se tiene que

$$
I=\frac{4}{\pi^{2}} \int_{0}^{\infty} \frac{z}{\sinh z} d z
$$

al sustituir las expresiones (5.1) y (5.2) en esta última integral se tiene

$$
\begin{aligned}
I & =\frac{2}{\pi^{2}} \int_{0}^{\infty} z \frac{\cosh \left(\frac{1}{2} z\right)}{\sinh \left(\frac{1}{2} z\right)} d z-\frac{2}{\pi^{2}} \int_{0}^{\infty} z \frac{\sinh \left(\frac{1}{2} z\right)}{\cosh \left(\frac{1}{2} z\right)} d z \\
& =\frac{4}{\pi^{2}}\left\{\lim _{z \rightarrow \infty} z \ln \left[\tanh \left(\frac{z}{2}\right)\right]-\lim _{z \rightarrow 0} z \ln \left[\tanh \left(\frac{z}{2}\right)\right]-\int_{0}^{\infty} \ln \left[\tanh \left(\frac{z}{2}\right)\right] d z\right\}
\end{aligned}
$$

los dos límites respectivamente tienden a cero, por lo tanto

$$
\begin{aligned}
I & =\frac{4}{\pi^{2}} \int_{0}^{\infty} \ln \left(\operatorname{coth}\left(\frac{z}{2}\right)\right) d z=\frac{4}{\pi^{2}} \int_{0}^{\infty} \ln \left(\frac{e^{\frac{1}{2} z}+e^{-\frac{1}{2} z}}{e^{\frac{1}{2} z}-e^{-\frac{1}{2} z}}\right) d z \\
& =\frac{4}{\pi^{2}} \int_{0}^{\infty} \ln \left(\frac{e^{z}+1}{e^{z}-1}\right) d z=\frac{4}{\pi^{2}} \frac{\pi^{2}}{4}=1,
\end{aligned}
$$

aquí se usó la expresión (18.101) dada en Spiegel et al. (2008).

Ahora se verifica que la función dada en (2.3) es la función de distribución, para ello se plantea

$$
I(x)=2\left(\frac{1}{\beta \pi}\right)^{2} \int_{-\infty}^{x}(u-\mu) \operatorname{csch}\left(\frac{1}{\beta}(u-\mu)\right) d u
$$

puesto que $\beta>0$, haciendo

$$
z=\frac{1}{\beta}(u-\mu) \rightarrow d z=\frac{1}{\beta} d u
$$

se llega a

$$
\begin{aligned}
I(x) & =\frac{2}{\pi^{2}} \int_{-\infty}^{\frac{x-\mu}{\beta}} z \operatorname{csch}(z) d z=\frac{2}{\pi^{2}} \int_{-\infty}^{\frac{x-\mu}{\beta}} \frac{z}{\sinh z} d z \\
& =\frac{2}{\pi^{2}}\left[\int_{-\infty}^{0} \frac{z}{\sinh z} d z+\int_{0}^{\frac{x-\mu}{\beta}} \frac{z}{\sinh z} d z\right]
\end{aligned}
$$

la última función que aparece en la integral como ya se dijo representa la forma simple y por la propiedad de simetría respecto al origen, se tiene que

$$
I(x)=\frac{1}{2}+\frac{2}{\pi^{2}} \int_{0}^{\frac{x-\mu}{\beta}} \frac{z}{\sinh z} d z
$$


al sustituir las expresiones (5.1) y (5.2) en esta última integral se tiene

$$
\begin{aligned}
I(x)= & \frac{1}{2}+\frac{1}{\pi^{2}} \int_{0}^{\frac{x-\mu}{\beta}} z \frac{\cosh \left(\frac{1}{2} z\right)}{\sinh \left(\frac{1}{2} z\right)} d z-\frac{1}{\pi^{2}} \int_{0}^{\frac{x-\mu}{\beta}} z \frac{\sinh \left(\frac{1}{2} z\right)}{\cosh \left(\frac{1}{2} z\right)} d z \\
= & \frac{1}{2}+\frac{2}{\pi^{2}}\left\{\frac{x-\mu}{\beta} \ln \left[\tanh \left(\frac{1}{2} \frac{x-\mu}{\beta}\right)\right]-\lim _{z \rightarrow 0} z \ln \left[\tanh \left(\frac{z}{2}\right)\right]\right\} \\
& -\frac{2}{\pi^{2}} \int_{0}^{\frac{x-\mu}{\beta}} \ln \left[\tanh \left(\frac{z}{2}\right)\right] d z
\end{aligned}
$$

como ya se mencionó este límite tiende a cero, por lo tanto

$$
I(x)=\frac{1}{2}+\frac{2}{\pi^{2}}\left\{\frac{x-\mu}{\beta} \ln \left[\tanh \left(\frac{1}{2} \frac{x-\mu}{\beta}\right)\right]-\int_{0}^{\frac{x-\mu}{\beta}} \ln \left[\tanh \left(\frac{z}{2}\right)\right] d z\right\},
$$

$\mathrm{Al}$ realizar el cambio de variable

$$
v=\tanh \left(\frac{z}{2}\right) \rightarrow d v=\frac{1}{2}\left[1-\tanh ^{2}\left(\frac{z}{2}\right)\right] d z
$$

se llega a

$$
I(x)=\frac{1}{2}+\frac{2}{\pi^{2}}\left\{\frac{x-\mu}{\beta} \ln \left[\tanh \left(\frac{1}{2} \frac{x-\mu}{\beta}\right)\right]+\int_{0}^{\tanh \left(\frac{1}{2} \frac{x-\mu}{\beta}\right)} \frac{2 \ln v}{v^{2}-1} d v\right\} .
$$

Puesto que

$$
\frac{2}{v^{2}-1}=\frac{1}{v-1}-\frac{1}{v+1}
$$

sea

$$
\begin{aligned}
I_{1}(x) & =\frac{2}{\pi^{2}} \int_{0}^{y} \frac{2 \ln v}{v^{2}-1} d v=\frac{2}{\pi^{2}} \int_{0}^{y}\left(\frac{1}{v-1}-\frac{1}{v+1}\right) \ln v d v \\
& =\frac{2}{\pi^{2}}\left[\int_{0}^{1} \frac{\ln v}{v-1} d v-\int_{y}^{1} \frac{\ln v}{v-1} d v-\int_{0}^{1} \frac{\ln v}{v+1} d v+\int_{y}^{1} \frac{\ln v}{v+1} d v\right] \\
& =\frac{2}{\pi^{2}}\left[\frac{\pi^{2}}{6}+\int_{1}^{y} \frac{\ln v}{v-1} d v+\frac{\pi^{2}}{12}-\int_{1}^{y} \frac{\ln v}{v+1} d v\right] \\
& =\frac{1}{2}-\frac{2}{\pi^{2}}\left[I_{2}(y)+I_{3}(y)\right],
\end{aligned}
$$

donde $y=\tanh \left(\frac{1}{2} \frac{x-\mu}{\beta}\right)$ aquí se usaron las expresiones (18.91) y (18.92) dadas en citeasnounSch. Empleando la expresión (27.7.1) dada en Abramowitz \& Stegun (1965), se tiene que

$$
I_{2}(y)=-\int_{1}^{y} \frac{\ln v}{v-1} d v=\operatorname{Li}_{2}(y)
$$

Comunicaciones en Estadística, diciembre 2010, Vol. 3, No. 2 
donde $\operatorname{Li}_{2}(\cdot)$ denota la función dilogarítmica, para realizar la segunda integral en (5.3) se emplea partes

$$
I_{3}(y)=\int_{1}^{y} \frac{\ln v}{v+1} d v=\left.\ln v \ln (v+1)\right|_{v=1} ^{v=y}-\int_{1}^{y} \frac{1}{v} \ln (v+1) d v
$$

y haciendo el cambio de variable $w=v+1$, se llega a

$$
\begin{aligned}
I_{3}(y) & =\ln y \ln (y+1)+\int_{1+y}^{2} \frac{\ln w}{w-1} d w \\
& =\ln y \ln (y+1)+\int_{1}^{2} \frac{\ln w}{w-1} d w-\int_{1}^{1+y} \frac{\ln w}{w-1} d w \\
& =\ln y \ln (y+1)+\frac{\pi^{2}}{12}+\operatorname{Li}_{2}(1+y)=\operatorname{Li}_{2}(y)-\frac{1}{2} \operatorname{Li}_{2}\left(y^{2}\right),
\end{aligned}
$$

en esta última integral se usaron las expresiones (18.93) y (27.7.6) dadas en Spiegel et al. (2008) y Abramowitz \& Stegun (1965), respectivamente. Luego,

$$
I_{1}(y)=\frac{1}{2}-\frac{2}{\pi^{2}}\left[2 \operatorname{Li}_{2}(y)-\frac{1}{2} \operatorname{Li}_{2}\left(y^{2}\right)\right] .
$$

Por lo tanto,

$$
I(x)=1-\frac{1}{\pi^{2}}\left\{4 \operatorname{Li}_{2}(y)-\operatorname{Li}_{2}\left(y^{2}\right)-2 \frac{x-\mu}{\beta} \ln (y)\right\},
$$

al sustituir $y$, se obtiene la expresión dada en (2.3).

En este apartado se realizan las demostraciones de los teoremas presentados.

Demostración. (Teorema 2.1)

1. La función $f_{X}(x)$ es continua en $x=\mu$, ya que

$$
\lim _{x \rightarrow \mu} f_{X}(x)=2 \lim _{x \rightarrow \mu}\left(\frac{1}{\beta \pi}\right)^{2}(x-\mu) \operatorname{csch}\left(\frac{x-\mu}{\beta}\right)=\frac{2}{\pi^{2}} \frac{1}{\beta}=f_{X}(\mu) .
$$

2. Los puntos críticos de esta función, se obtienen

$$
\begin{aligned}
f_{X}^{\prime}(x) & =2\left(\frac{1}{\beta \pi}\right)^{2} \frac{\sinh \left(\frac{x-\mu}{\beta}\right)-\left(\frac{x-\mu}{\beta}\right) \cosh \left(\frac{x-\mu}{\beta}\right)}{\sinh ^{2}\left(\frac{x-\mu}{\beta}\right)} \\
& =2\left(\frac{1}{\beta \pi}\right)^{2}\left(\frac{1-\left(\frac{x-\mu}{\beta}\right) \operatorname{coth}\left(\frac{x-\mu}{\beta}\right)}{\sinh \left(\frac{x-\mu}{\beta}\right)}\right)
\end{aligned}
$$

Comunicaciones en Estadística, diciembre 2010, Vol. 3, No. 2 
por lo tanto, $f_{X}(x)$, tiene un punto crítico cuando

$$
\frac{x-\mu}{\beta} \operatorname{coth}\left(\frac{x-\mu}{\beta}\right)=1 \quad \Rightarrow \quad \tanh \left(\frac{x-\mu}{\beta}\right)=\frac{x-\mu}{\beta},
$$

es decir $x=\mu$. Evaluando la segunda derivada se tiene

$$
f_{X}^{\prime \prime}(x)=\frac{(x-\mu)\left(3+\cosh \left[\frac{-2}{\beta}(x-\mu)\right]\right)+2 \beta \sinh \left[\frac{-2}{\beta}(x-\mu)\right]}{\pi^{2} \beta^{4} \sinh ^{3} \frac{x-\mu}{\beta}}
$$

evaluando en esta expresión el límite cuando $x \rightarrow \mu$, se obtiene

$$
\begin{aligned}
f_{X}^{\prime \prime}(\mu) & =\lim _{x \rightarrow \mu} \frac{(x-\mu)\left(3+\cosh \left[\frac{-2}{\beta}(x-\mu)\right]\right)+2 \beta \sinh \left[\frac{-2}{\beta}(x-\mu)\right]}{\pi^{2} \beta^{4} \sinh ^{3} \frac{x-\mu}{\beta}} \\
& =-\frac{2}{3 \pi^{2} \beta^{3}}
\end{aligned}
$$

como $f_{X}^{\prime \prime}(\mu)<0$ entonces $x=\mu$ es un máximo.

3. Para ver que es simétrica respecto a $\mu$, se debe verificar que

$$
f_{X}(\mu+x)=f_{X}(\mu-x), \quad \text { para todo } x .
$$

Nótese que

$$
\begin{aligned}
f_{X}(\mu-x) & =2\left(\frac{1}{\beta \pi}\right)^{2}(-x) \operatorname{csch}\left(\frac{1}{\beta}(-x)\right) \\
& =2\left(\frac{1}{\beta \pi}\right)^{2} x \operatorname{csch}\left(\frac{1}{\beta} x\right)=f_{X}(\mu+x)
\end{aligned}
$$

4. El eje $X$ es una asíntota horizontal de la función $f_{X}(x)$, pues

$$
\begin{aligned}
& \text { a) } \lim _{x \rightarrow \infty} f_{X}(x)=2 \lim _{x \rightarrow \infty}\left(\frac{1}{\beta \pi}\right)^{2}(x-\mu) \operatorname{csch}\left(\frac{x-\mu}{\beta}\right) \\
& =\frac{2}{\beta \pi^{2}} \lim _{u \rightarrow \infty} u \operatorname{csch}(u)=0 \\
& \text { b) } \lim _{x \rightarrow-\infty} f_{X}(x)=2 \lim _{x \rightarrow-\infty}\left(\frac{1}{\beta \pi}\right)^{2}(x-\mu) \operatorname{csch}\left(\frac{x-\mu}{\beta}\right) \\
& =\frac{2}{\beta \pi^{2}} \lim _{u \rightarrow-\infty} u \operatorname{csch}(u)=0
\end{aligned}
$$

Comunicaciones en Estadística, diciembre 2010, Vol. 3, No. 2 
Demostración. (Teorema 2.2)

En general, como la función de densidad es simétrica con respecto a $\mu$, todos los momentos centrales de orden impar son iguales a cero.

Los momentos centrales de orden par se establecen como sigue

$$
\mu_{2 m}=2\left(\frac{1}{\beta \pi}\right)^{2} \int_{-\infty}^{\infty}(u-\mu)^{2 m+1} \operatorname{csch}\left(\frac{1}{\beta}(u-\mu)\right) d u,
$$

puesto que $\beta>0$, haciendo

$$
z=\frac{1}{\beta}(u-\mu) \rightarrow d z=\frac{1}{\beta} d u
$$

se llega a

$$
\mu_{2 m}=\frac{2}{\beta \pi^{2}} \int_{-\infty}^{\infty}(\beta z)^{2 m+1} \operatorname{csch}(z) d z=2\left(\frac{\beta^{m}}{\pi}\right)^{2} \int_{-\infty}^{\infty} \frac{z^{2 m+1}}{\sinh z} d z
$$

por la propiedad de simetría respecto al origen, se tiene que

$$
\mu_{2 m}=4\left(\frac{\beta^{m}}{\pi}\right)^{2} \int_{0}^{\infty} \frac{z^{2 m+1}}{\sinh z} d z
$$

usando la expresión (18.115) dada en Spiegel et al. (2008), se obtiene que

$$
\mu_{2 m}=\frac{2^{2(m+1)}-1}{2^{2 m-1}}\left(\frac{\beta^{m}}{\pi}\right)^{2} \Gamma(2(m+1)) \sum_{k=1}^{\infty} \frac{1}{k^{2(m+1)}},
$$

y empleando la expresión (21.35) dada en Spiegel et al. (2008), se llega a

$$
\mu_{2 m}=2(-1)^{m} \frac{2^{2(m+1)}-1}{m+1}(\beta \pi)^{2 m} B_{2(m+1)},
$$

donde, $B_{k}$ denota el $k$-ésimo número de Bernoulli, los cuales pueden encontrarse de la siguiente expansión de Taylor.

$$
\frac{x}{e^{x}-1}=1-\frac{1}{2} x+\sum_{m=1}^{\infty} B_{2 m} \frac{x^{2 m}}{(2 m) !}, \quad|x|<2 \pi,
$$

En la Tabla 5 se dan los primeros números de Bernoulli

\begin{tabular}{|c|cccccccccc|}
\hline$m$ & 1 & 2 & 3 & 4 & 5 & 6 & 7 & 8 & 9 & 10 \\
\hline$B_{2 m}$ & $\frac{1}{6}$ & $-\frac{1}{30}$ & $\frac{1}{42}$ & $-\frac{1}{30}$ & $\frac{5}{66}$ & $-\frac{691}{2730}$ & $\frac{7}{6}$ & $-\frac{3617}{510}$ & $\frac{43867}{798}$ & $-\frac{174611}{330}$ \\
\hline
\end{tabular}

Tabla 5: Números de Bernoulli

Reemplazando en 5.5 los respectivos valores de $B_{k}$ se obtienen las expresiones (2.6) $\mathrm{y}(2.7)$. 
A continuación se presentan los valores tabulados de la distribución.

\begin{tabular}{|c|c|c|c|c|c|c|c|c|c|c|}
\hline$z^{*}$ & 0.00 & 0.01 & 0.02 & 0.03 & 0.04 & 0.05 & 0.06 & 0.07 & 0.08 & 0.09 \\
\hline 0.0 & 0.5000 & 0.5045 & 0.5090 & 0.5135 & 0.5180 & 0.5225 & 0.5270 & 0.5315 & 0.5359 & 0.5404 \\
\hline 0.1 & 0.5449 & 0.5494 & 0.5538 & 0.5583 & 0.5627 & 0.5671 & 0.5715 & 0.5759 & 0.5803 & 0.5847 \\
\hline 0.2 & 0.5891 & 0.5934 & 0.5977 & 0.6021 & 0.6064 & 0.6107 & 0.6149 & 0.6192 & 0.6234 & 0.6276 \\
\hline 0.3 & 0.6318 & 0.6360 & 0.6401 & 0.6443 & 0.6484 & 0.6525 & 0.6565 & 0.6606 & 0.6646 & 0.6686 \\
\hline 0.4 & 0.6726 & 0.6765 & 0.6804 & 0.6843 & 0.6882 & 0.6921 & 0.6959 & 0.6997 & 0.7034 & 0.7072 \\
\hline 0.5 & 0.7109 & 0.7146 & 0.7182 & 0.7218 & 0.7254 & 0.7290 & 0.7325 & 0.7360 & 0.7395 & 0.7429 \\
\hline 0.6 & 0.7464 & 0.7497 & 0.7531 & 0.7564 & 0.7597 & 0.7630 & 0.7662 & 0.7694 & 0.7726 & 0.7757 \\
\hline 0.7 & 0.7788 & 0.7819 & 0.7850 & 0.7880 & 0.7910 & 0.7939 & 0.7969 & 0.7998 & 0.8026 & 0.8054 \\
\hline 0.8 & 0.8082 & 0.8110 & 0.8138 & 0.8165 & 0.8191 & 0.8218 & 0.8244 & 0.8270 & 0.8296 & 0.8321 \\
\hline 0.9 & 0.8346 & 8371 & 0.8395 & 0.8419 & 0.8443 & 0.8466 & 0.8490 & 0.8512 & 0.8535 & 0.8558 \\
\hline 1.0 & 0.8580 & 8601 & 0.8623 & 0.8644 & 0.8665 & 0.8686 & 0.8706 & 0.8727 & 0.8746 & 0.8766 \\
\hline 1.1 & 0.8785 & 8805 & 0.8823 & .8842 & 0.8860 & 0.8879 & 0.8896 & 0.8914 & 0.8931 & 0.8948 \\
\hline 1.2 & 8965 & 8982 & 8998 & 9015 & 0.9031 & 0.9046 & 0.9062 & 0.9077 & 0.9092 & 0.9107 \\
\hline 1.3 & 9122 & 9136 & 9150 & .9164 & 0.9178 & 0.9192 & 0.9205 & 0.9218 & 0.9231 & 0.9244 \\
\hline 1.4 & 0.9257 & 9269 & .9281 & 0.9293 & 0.9305 & 0.9317 & 0.9328 & 0.9340 & 0.9351 & 0.9362 \\
\hline 1.5 & 0.9373 & 0.9383 & 0.9394 & 0.9404 & 0.9414 & 0.9424 & 0.9434 & 0.9444 & 0.9453 & 0.9463 \\
\hline 1.6 & 0.9472 & 0.9481 & 0.9490 & 0.9499 & 0.9507 & 0.9516 & 0.9524 & 0.9533 & 0.9541 & 0.9549 \\
\hline 1.7 & 0.9557 & 0.9564 & 0.9572 & 0.9579 & 0.9587 & 0.9594 & 0.9601 & 0.9608 & 0.9615 & 0.9622 \\
\hline 1.8 & 0.9628 & 0.9635 & 0.9641 & 0.9648 & 0.9654 & 0.9660 & 0.9666 & 0.9672 & 0.9678 & 0.9684 \\
\hline 1.9 & 0.9689 & 0.9695 & 0.9700 & 0.9706 & 0.9711 & 0.9716 & 0.9721 & 0.9726 & 0.9731 & 0.9736 \\
\hline 2.0 & 0.9741 & 0.9745 & 0.9750 & 0.9754 & 0.9759 & 0.9763 & 0.9767 & 0.9772 & 0.9776 & 0.9780 \\
\hline 2.1 & 0.9784 & 0.9788 & 0.9792 & 0.9795 & 0.9799 & 0.9803 & 0.9806 & 0.9810 & 0.9813 & 0.9817 \\
\hline 2.2 & 0.9820 & 0.9823 & 0.9827 & 0.9830 & & 0.9836 & 0.9839 & 0.9842 & & 0.9848 \\
\hline 2.3 & & & & & & & & 0.9 & 0.98 & 0.9874 \\
\hline 2.4 & 0.98 & & & & 0.9 & 0.9 & 0.9 & 0.9891 & 0.9893 & 0.9895 \\
\hline 2.5 & 0.9897 & 99 & .9901 & 0.9903 & 0.9 & 0.9906 & 0.9908 & 0.9910 & 0.9912 & 0.9913 \\
\hline 2.6 & 0.9915 & 9916 & 9918 & .9920 & 0.9 & 0.9923 & 0.9924 & 0.9925 & 0.9 & 0.9928 \\
\hline 2.7 & 9930 & & 9932 & .9933 & 0.9 & 0.9936 & 0.9937 & 0.9938 & 0.9940 & 0.9941 \\
\hline 2.8 & 0.9942 & 0.9943 & 0.9944 & 0.9945 & 0.9946 & 0.9947 & 0.9948 & 0.9949 & 0.9950 & 0.9951 \\
\hline 2.9 & 0.9952 & 9953 & 0.9954 & 0.9955 & 0.9956 & 0.9956 & 0.9957 & 0.9958 & 0.9959 & 0.9960 \\
\hline 3.0 & 0.9960 & 0.9961 & 0.9962 & 0.9963 & 0.9963 & 0.9964 & 0.9965 & 0.9965 & 0.9966 & 0.9967 \\
\hline 3.1 & 0.9967 & 0.9968 & 0.9969 & 0.9969 & 0.9970 & 0.9970 & 0.9971 & 0.9972 & 0.9972 & 0.9973 \\
\hline 3.2 & 0.9973 & 0.9974 & 0.9974 & 0.9975 & 0.9975 & 0.9976 & 0.9976 & 0.9977 & 0.9977 & 0.9977 \\
\hline 3.3 & 0.9978 & 0.9978 & 0.9979 & 0.9979 & 0.9980 & 0.9980 & 0.9980 & 0.9981 & 0.9981 & 0.9981 \\
\hline 3.4 & 0.9982 & 9982 & .9983 & 0.9983 & 0.9983 & 0.9984 & 0.9984 & 0.9984 & 0.9984 & 0.9985 \\
\hline 3.5 & 0.9985 & 0.9985 & 0.9986 & 0.9986 & 0.9986 & 0.9986 & 0.9987 & 0.9987 & 0.9987 & 0.9987 \\
\hline 3.6 & 0.9988 & 0.9988 & .998 & 88 & & & & & 0.9990 & 0.9990 \\
\hline 3.7 & & & & & & & & & & 0.9992 \\
\hline 3.8 & 0.9992 & & & & & & & & & 0.9993 \\
\hline 3.9 & & & & & & & & & & 0.9994 \\
\hline 4.0 & & & & & & & & & & 0.9995 \\
\hline 4.1 & & & & & & & & & & 0.9996 \\
\hline 4.2 & & & & & & & & & & 0.9997 \\
\hline 4.3 & & & & & & & & & & 0.9997 \\
\hline 4.4 & & & & & & & & & 0.9998 & 0.9998 \\
\hline 4.5 & 0 & & 0.99 & 0.99 & 0.99 & 0.9998 & 0.9998 & 0.9998 & 0.9998 & 0.9998 \\
\hline 4.6 & .9998 & 9998 & 0.9998 & 0.9998 & 0.9998 & 0.9999 & 0.9999 & 0.9999 & 0.9999 & 0.9999 \\
\hline 4.7 & 0 & 0.9999 & 0.9999 & 0.9999 & 0.9999 & 0.9999 & 0.9999 & 0.9999 & 0.9999 & 0.9999 \\
\hline 4.8 & 0 & 0.99 & 0.99 & 0.99 & 0.99 & 0.9999 & 0.9999 & 0.9999 & 0.9999 & 0.9999 \\
\hline 4.9 & 0. & & 0.99 & 0.99 & 0.99 & 0.9999 & 0.9999 & 0.9999 & 0.9999 & 0.9999 \\
\hline 5.0 & 0.9999 & 0.9999 & 0.9999 & 0.9999 & 0.9999 & 0.9999 & 0.9999 & 0.9999 & 0.9999 & 0.9999 \\
\hline
\end{tabular}

Comunicaciones en Estadística, diciembre 2010, Vol. 3, No. 2 